\title{
A CASE OF MULTIPLE MYCOTIC ANEURYSMS OF THE FIRST PART OF THE AORTA.
}

\author{
By John M ${ }^{\mathrm{c} C R A E}$, M.B., L.R.C.P.(Lond.), Lecturer in Pathology, \\ M'Gill University, Montreal.
}

From the J. H. R. Molson Pathological Laboratory.

Tre subject of this report died on board ship near Montreal, and the body was brought to port for autopsy and burial; the history of the case is somewhat obscure. He was a ship surgeon, æt. about 36 , and had suffered for some years with "heart disease" and "stomach trouble." At 28 he had an attack of rheumatism, and was for many years addicted to the immoderate use of alcohol, drinking steadily, and at times to excess. A month before his death he bad much cardiac pain, and was unfit for work, although he kept at his duties. On his last voyage he was confined to bed most of the time, and one day, while lying in his berth conversing with a friend in a near-by cabin, he suddenly stopped speaking and was found dead.

The autopsy was nade 72 hours after death, and bacteriological examination was not made on that account. The anatomical diagnosis was as follows:-

Clironic sclerotic aortic and mitral endocarditis, with mitral stenosis, and evident aortic insufficiency; five small aneurysms of ascending aorta with acute vegetative endarteritis of the surrounding intima; perforation of one of these with hæmopericardium; acute fibrinous pericarditis; chronic diffuse myocarditis ; dilatation and hypertrophy of heart ; induration of all abdominal organs; arterio-sclerosis; œdema of lungs; bilateral hydrothorax; nutmeg liver ; chronic catarrhal gastritis; œdema of pia.

The picture is therefore that of recurrent valvular disease of the heart, with myocardial change and irreparable breaking of compensation, and, as a terminal event, aneurysmal rupture of the aorta.

On opening the thorax the pericardium was seen to occupy a large space, and, on opening it, blood and clot were found to the amount of $20 \mathrm{oz}$. The entire surface of the pericardium was covered by recent fibrin, and in places the heart had to be separated by force from the parietal pericardium, which was thickened throughout, the loose tissue on its mediastinal surface being œedematous. The fibrin was everywhere interspersed with blood clot, but the source of the hæmorrhage was not yet apparent. Smears from the pericardium showed blood cells, but no organisms.

The heart weighed 1170 grms. The valves measured as follows:-tricuspid, $10 \mathrm{~cm}$. ; mitral, $7 \mathrm{~cm}$.; pulmonary, $8.25 \mathrm{~cm}$.; aortic, $7.5 \mathrm{~cm}$. There was a slight mitral stenosis, the valve being fused, calcified, eroded, but without any recent vegetations. The aortic valves were calcified, evidently incompetent, the edges piled up in calcareous masses that resembled fresh vegetations, but were stony hard to the finger.

On the aorta, as one looked at it opened up along the anterior aspect, five openings were seen, the lowest an inch above the free edge of the posterior cusp, and measuring $0.5 \mathrm{~cm}$. in diameter, having around it a recent hedge of 
red vegetations that projected $3 \mathrm{~mm}$. from the surface. A probe passed through this into the pericardium, and this was the site of rupture. Four others appeared farther up on the aorta, all smaller than the first, two showing a short split of the aorta wall continuous with the opening. These slits were as clear-cut and definite as if made with a sharp knife. Vegetations of the same nature as before described appeared on the edges of all. One of these aneurysms was cut into for microscopic examination, and proved to be a little sac continuous with the vessel lumen through the broken intima and media, the cellular tissue of the mediastinum evidently forming the wall of it.

In this tissue there was a little blood effused, and the inner surface of the sac appeared indistinct and rough. It seemed a weak barrier to interpose against the pressure of the blood so near the heart. Upon two of the

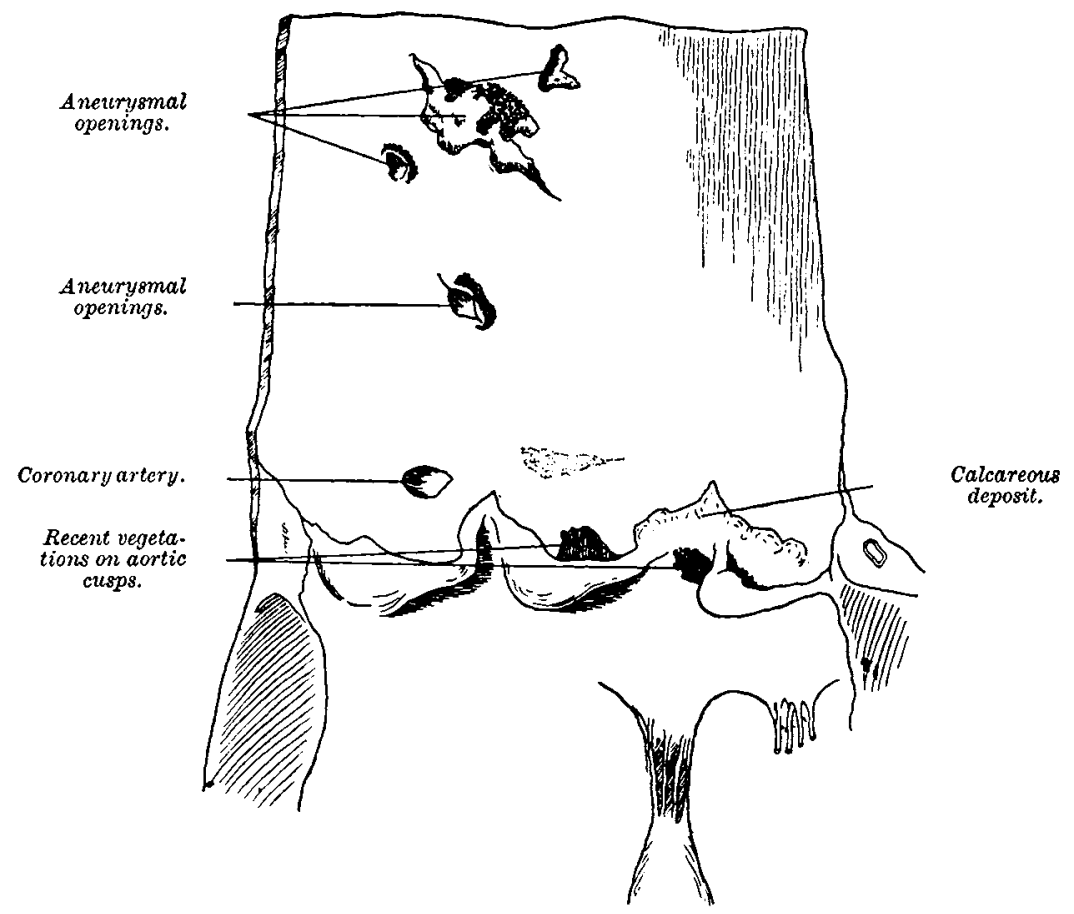

Diagrammatic representation of aorts, opened up, with four aneurysmal openings.

openings, at the edges, there was a yellow patch on the intima that suggested arterio-sclerosis. The heart muscle was brown and flabby; microscopic examination showed much fragmentation of fibres, but fat was not demonstrated in the cells.

Microscopic examination of one of these openings in the aorta (not one showing the sharp, incision-like tears) showed that, almost up to the point of rupture, the media consisted of normal tissue, the elastic fibres being intact. The appearance of the sac indicated that, at the point of rupture, the blood had effused and impinged against the broken edge, forcing asunder the fibres as when one blows against the pages of a loosely held book and forces them apart in groups of two and three pages each. The cellular tissue of the adventitia bounded the aneurysmal cavity, which had many bays and inlets filled up by more or less organised blood clot. The wall and the partially organised fibrin were densely infiltrated by leucocytes and cells of the type 
associated with inflammatory reaction, though nowhere to the extent that is seen in early abscess formation. In parts the wall of the sac was densely fibrous, and in the centre of the sac was a mass of fibrin which was loosely joined at one end only to the rough sac wall. No new-formed intima was seen in the sac, nor calcareous deposit in the vicinity. Sections stained for bacteria showed on the superficial parts of the sections a few bacilli and cocci, indicating that the organisms of decomposition were scanty despite the time that had elapsed before autopsy. In the infiltrated tissues at the bottom of the sac were numerous micrococci, both in the cells and between them. These were well shown in sections stained by Gram's method. At this point it must not be forgotten that an infective pericarditis was present, and with an acute inflammatory process at work on both sides of the sac wall it is easy to understand the final weakening of the wall and the consequent perforation.

The case falls under the category of parasitic or mycotic (i.e. bacterial) aneurysms, as described by Eppinger $\left({ }^{1}\right)$; and although it partakes evidently of the nature of those cases usually called "false aneurysms," it is equally true of the nature of "aneurysma disecans." Though there is little actual burrowing of the blood stream, the layers of the media are separated at the edge of the aneurysmal sac, and more extensive dissection might accidently occur by - a further exertion of the same forces that have already been at work.

The mode of development of this type of aneurysm is obscure, and Eppinger ( $\left.{ }^{1}\right)$ and Bostroem ( ${ }^{2}$, as well as Thoma $\left({ }^{3}\right)$, all of whom have discussed the subject at some length, are not in complete agreement in their views. Two possibilities are to be cousidered: (a) Has there been a primary local infection of the intima with erosion and ulceration, the ulceration spreading into the media and resulting in an infiltration of aortic blood between the layers of the media, and finally completely through the media into the adventitia? or (b) has the infective agent, vid the vasa vasorum, set up a primary inflammation of the media (an acute mesarteritis), with resulting infiltration and degeneration of the elastica and other tissues of the involved area, the intima being only secondarily involved; and, as a result of this 'softening and weakening of its underlying support, the intima has given way with the production of a dissecting aneurysm? Eppinger's own words are: "By a mycotic embolic thrombosis (i.e. in one of the vasa vasorum) there occurs an acute peri- and mesarteritis, leading to complete solution of continuity of the elastica and damage to the intima, and finally to the bulging of the recently inflamed but yet resistant remaining tissue (e.g. adventitia) to form an aneurysm." Eppinger further insists upon the multiplicity of the lesions within a small radius being the strongest evidence of its embolic or thrombotic origin.

In the case here described the former of the two modes of causation may be dismissed, for the following reasons:-

1. If this very advanced condition of mycotic aneurysm originated from the intima we should have more frequently evidence of the slighter condition, namely, of infective, ulcerative process confined to 
the intima, but anything like positive indications of an infective process limited to the aortic intima are of great rarity, though infective endocarditis and the presence of bacteria in the blood stream are common conditions. The aortic intima is remarkably insusceptible to direct infection. Further, Eppinger notes the presence of bacteriaholding fibrin on the intimal surface of the aorta close to or even in the neck of the sac, and this was not seen in the present case.

2. In advanced arterio-sclerosis, on the other hand, we do encounter disease of the intima, leading frequently to extensive ulceration (non-infective, atheromatous ulcers). This process may also involve the media, but under these conditions it is remarkable that we do not find the development of dissecting aneurysm, which is as rare as that of ulcerating arterio-sclerosis is common.

It would seem, therefore, that in this case we have to deal with an infective mesarteritis, or, more correctly, mesaortitis.

But, admitting this, there remain certain difficulties before we can clearly understand the whole course of the case. There is present a moderate grade of sclerosis, with thickening of the intima. Has this played any part in the production of the aneurysm? What is the, nature of the sharply cut slits of the intima seen here, as in so many dissecting aneurysms? Does the explanation given for the development of this infective form apply to dissecting aneurysms in general, or have we to recognise at least two orders of aneurysm of this nature? These questions are closely allied, and must be discussed, if briefly.

As Adami ( $\left.{ }^{4}\right)$ points out, it is noteworthy that dissecting aneurysms are always associated with arterio-sclerosis; not with the extreme grades, but with a moderate grade, with hyalin rather than with fatty and calcareous changes in the intima; and he has suggested that, in the more extreme grades, the thickened intima is necrotic, and that when it ulcerates and erodes the dead matter may be washed loose; whereas in hyalin change the tissue is still living, and in organic connection with the underlying layers. But such hyalin material is brittle, and when a blow or an extra strain from sudden increase of pressure occurs there is a rupture, not only of the intima, but also of the organically connected media, the split extending into that coat, and thus leading to the production of a dissecting aneurysm. Such a brittle condition may be the explanation of the sharp-cut cleavage of the intima which is found in these cases. Cuts such as those described in this case strongly recall the sharp splits of the scalp that can be produced even by a blunt instrument, because the scalp is in a tense state over the skull. It may be that the aorta is stretched over the column of blood (if one may use those terms), and the sharp incision-like cut is produced by the tension. These transverse slits, looking like incisions, are of such frequent occurrence with dissecting aneurysms that there is evidently a relationship between the phenomena. 
Some recent studies raise the question whether thickening of the intima be an essential forerunner of the production of linear slits in the intima. More than one observer has noted what must be the cicatrices of such slits in the first part of the aorta, namely, fine linear scars in the apparently otherwise healthy intima. Certain cases quoted by Tolot and Sarvonat $\left(^{5}\right)$ indicate that intimal change is not an essential: their own case is a man of 68 , whose atheromatous aorta ruptured in a place that was apparently healthy; they cite six cases in which rupture of the aorta occurred without any trace of atheroma, three of them occurring in males of 20,22 , and 25 years of age, and one in a female of 23 years. It may be useful to refer to these cases in short detail. The first, æt. 42, was paraplegic; the second, xt. 22 , was apparently healthy; the third, a male of 25 , was apparently healthy, but the aorta near the rupture was thin; the fourth, a woman of 23, had had typhoid fever, and an old nephritis; the fifth, reported by Brouardel and Vibert, had had typhoid fever; at autopsy it was found that the aorta had ruptured twice, the one rupture being $36 \mathrm{~mm}$. below the left carotid, the other $3 \mathrm{~cm}$. above the diaphragm; the media was found "amorphous and granular," and the aorta wall was thin. It will be noticed that in the first, fourth, and fifth cases there is a suggestion of cause for the mesarteritis that we must suppose has existed: one mentally assumes that there must be change of the media, although visible intimal change does not occur. If the healthy intima, or the healthy artery, were liable to slit we might expect to find these cicatrices (referred to above), and dissecting aneurysms, at all periods of life. As a matter of fact, both conditions are found almost entirely after the age of thirty, after the age, that is, when sclerosis begins to show itself as a marked feature.

Professor Adami pointed out to me, when studying the present case, that he had not, in his article on dissecting aneurysms (referred to), taken sufficiently into account the part which diseases of the media might play. In his advanced cases of long duration, indications of acute local degenerative changes in the media were of necessity wanting, whereas here they were definitely present. It would not be safe to say that all dissecting aneurysms are of similar origin, because perhaps a majority of the cases occur without any signs of concurrent infection. But it seems reasonable to state that there is a group of mycotic aneurysms in which the main rôle is played by local acute infective mesarteritis, where degenerative changes in the intima-such as appear in the present case-are wholly secondary, though, it may be, auxiliary.

The question whether local degenerative change in the media is an essential factor in the production of the non-mycotic dissecting aneurysms remains an open one. One has but to read the remarkably full discussion upon syphilitic aortitis and its relation to aneurysm, at the meeting of the German Pathological Society, to recognise how 
important a part this is now regarded as playing in the development of aneurysms in general. In opening that discussion Chiari $\left(^{6}\right)$ laid down that there are to be recognised two main forms of "aortitis": the more usual is that in which the intima is alone or primarily affected, undergoing thickening and atheroma; the other is that in which there is characteristically a mesaortitis, with secondary affection of the intima; and this mesaortitis is most often of syphilitic origin. Heiberg in Norway, and Laveran in France, independently noted its existence in 1877, and noted that it is a factor in the production of aneurysms in the syphilitic. Since then a series of pupils of Heller in Kiel have brought forward case after case in which they have found miliary gummata, with other processes of a syphilitic nature, in the middle coat of the aorta in cases of aneurysm. Chiari himself found a mesaortitis of this type present in sixteen out of twenty-seven cases with an acknowledged syphilitic history, and four of these sixteen showed aneurysms. In his remarks closing the discussion he expresses the wish that studies be made in connection with other infectious diseases, to determine whether some of them do not cause a similar mesaortitis.

Such infective mesaortitis or mesarteritis has been recognised ever since 1829 , when Andral $\left({ }^{7}\right)$ described a case showing half a dozen abscesses under the intima of the aorta, each containing pus like that of an ordinary suppurative phlegmon. Among others, Virchow $\left({ }^{8}\right.$ ) has noted abscess production in the wall (in a case of endaortitis), while Rokitansky $\left({ }^{9}\right)$ is said to have been the first to note the relationship of such abscesses to the production of an aneurysm by the bursting of an intra-parietal abscess into the lumen of the aorta.

The evidence, therefore, is adequate to prove the relationship of acute mesaortitis as well as of syphilitic mesaortitis to aneurysm production; and although, from the very nature of the process, it is impossible for us to make sure that dissecting aneurysms in general are due to these conditions, we are at least justified by analogy in assuming that such is probably the case.

I should add, in conclusion, that I have made a series of sections through the aorta in my specimen to determine whether there might not be localised areas of small-celled infiltration in the middle coat showing the earlier stage of infection without aneurysm production, but $I$ have not found them.

In brief recapitulation of the case here given, the evidence of the mycotic nature of the lesion rests upon-

1. The coexistence of recurrent endocarditis.

2. The fact that the sites of aneurysms are multiple within a small area.

3. The great degree of small-celled infiltration, which is scarcely explicable upon the basis of mere repair. 
4. The likelihood that the recent vegetations are an indication of continued bacterial activity.

5. The actual presence of micrococci in large number in the walls of the cavity.

The sequence of events in the case may be supposed to have been as follows:-

1. Infected thrombosis of vessel or collateral group of vessels supplying the ascending aorta.

2. Splitting of the wall of the aorta, with subsequent rounding of edges, or else simple ulceration from mesarteritis.

3. Contemporaneous formation of aneurysm.

4. Growth of fresh vegetations on edges of openings.

5. Further splitting of aorta from the peripheries of two of the aneurysmal openings, these again being sharp and incised looking.

6. Rupture of one of the aneurysms, with death.

\section{REFERENCES.}

1. Eppinger . . . . . . Arch. f. klin. Chir., Berlin, Bd. xxxv. S. 156.

2. Bostroem . . . . . . Deutsches Arch. f. hlin. Med., Leipzig, 1888, Bd. xlii. S. 1.

3. Thомa. . . . . . . Virchow's Archiv, 1888, Bd. cxi. S. 76 ; Ibid., 1888, Bd. cxii. S. 254 ; ILid., 1888, Bd. cxiii. S. 244.

4. AdaMi . . . . . . . Montreal Med. Journ., 1896, June and July.

5. Tolot et Sarvonat . . Rev. de méd., Paris, 1904, p. 840.

*6. Chiari and othens . . Verhandl. d. deutsch. path. Gesellsch., Berlin, 1903; Centralbl. f. Bahteriol.u. Parasitenk, Jena, 1904, Bd. xv., Erganzungsheft.

7. Andral . . . . . "Précis d'Anat. Pathologique," Paris, 1829, tome ii. p. 379.

*8. Virchow . . . . . . "Ges. Abhandl.," Berlin, 1879, S. 403.

9. RokitanskY . . . . . "Handbuch der Path. Anat.,"Wien, 1844, Bd. ii. S. 533.

* Not verified. 